\title{
Eugen Pfister
}

Der Politische Mythos als diskursive Aussage im digitalen Spiel

Ein Beitrag aus der Perspektive der Politikgeschichte

Erschienen in:

Junge, Thorsten/Clausen, Dennis (Hrsg.):

Digitale Spiele im Diskurs

Kultur- und

Sozialwissen-

schaften

Level 1: Herausforderungen und Problemfelder

\section{(c) (1) $(\Theta \Theta$}




\section{Der Politische Mythos als diskursive Aussage im digitalen Spiel}

Ein Beitrag aus der Perspektive der Politikgeschichte.

Eugen Pfister

\section{„Keep your Politics out of my Games."}

Eine Verbindung zwischen Computerspielen und Politik wird von vielen SpielerInnen aber auch von EntwicklerInnen häufig als Problem verstanden, welches es zu vermeiden gelte. Vor allem in tendenziell konservativen bis rechten Spieleforen findet sich häufig der trotzige Ausruf: „Keep your Politics out of my Games!'. ${ }^{1}$ Darauf angesprochen, ob ihre digitalen Spiele nicht auch eine politische Aussage hätten, reagieren die meisten EntwicklerInnen und vor allem MitarbeiterInnen der großen Spielevertriebe im Regelfall ablehnend. Häufig trifft man auf die Aussage, es seien doch „nur Spiele“ “. ${ }^{2}$ Vor Kurzem etwa erklärte Reggie Fils-Aime, Präsident von Nintendo of America in einem Interview im kanadischen Fernsehen CBC: „Making political statements are for other people to do. We want people to smile and have fun when they play our games. “' Die Ablehnung des „Politischen“, die auch im Kontext einer wachsenden Politikverdrossenheit innerhalb der europäischen und nordamerikanischen Bevölkerungen verstanden werden muss, ist vor allem Indiz für ein sehr restriktives und negatives populäres Politikverständnis. Dass der selbstgestellte Anspruch, unpolitischen Spaß zu garantieren, aber gar nicht umsetzbar ist, zeigt das folgende Beispiel. Julian Gerighty, Associate Creative Director von Tom Clancy: The Division (Massive Entertainment: SE 2016)

\footnotetext{
${ }^{1}$ Vergleiche dazu Christopher Franklin, "Keep Your Politics Out Of My Video Games" in errantsignal.com <http://www.errantsignal.com/blog/?p=582>. Ironischerweise wurde dieser Ruf nach dem Apolitischen insbesondere im Zuge der sogenannten „Gamergate“Bewegung laut, die wiederum enge Verbindungen zu Alt-Right-Gruppierungen und dem Entourage von Donald Trump aufweisen. Siehe dazu: Matt Lees, „, What Gamergate should have taught us about the 'alt-right' in theguardian.com (01.12.2016) <https://www.theguardian.com/technology/2016/dec/01/gamergate-alt-right-hatetrump $>$

${ }^{2}$ Vgl. dazu: „Die für die Komplexitätsreduktion in Kauf genommenen Verzerrungen und Vereinfachungen sozialer Realität zeugen indes auch von einer wenig entwickelten Vorstellung der Spielemacher von der sozialen Bedeutung ihrer Produkte" in Klimmt, 2009, 73. 3 Nachrichtensendung "The National“" (15. Juni 2017) in youtube.com $<$ https://www.youtube.com/watch?v=yzJ0P0c3MxM>
}

erklärte in einem Interview: „At the end of the day, it's a videogame, it's an entertainment product... There's no particularly political message with it." ${ }^{34}$ Aber: Die Handlung des Third-Person Shooters setzt kurz nach dem politischen Kollaps in New York ein. ${ }^{5}$ Die Infrastruktur ist im Anschluss an einen eine Pandemie auslösenden Terroranschlag zusammengebrochen. Polizei, Rettung und Müllabfuhr versagen ihren Dienst. Das Militär zieht sich aus der Stadt zurück und die US-Regierung muss auf paramilitärische Schläfer zurückgreifen, die mittels Gewalteinsatz für Ordnung sorgen. Ein Narrativ also, das auch nach einer engen Auslegung des Begriffs sehr deutlich eine „politische“ Aussage beinhaltet: die demokratisch gewählte US-amerikanische Regierung und die Stadtverwaltung von New York sind nicht in der Lage, mithilfe ihrer konventionellen exekutiven Mittel adäquat auf solch ein Bedrohungsszenario zu reagieren. Es ist aber eben nicht die Hintergrundstory allein - die theoretisch beim Spielen ignoriert werden könnte - es sind vor allem die Ästhetik eines zerstörten Manhattan und die Spielmechanik der Justiz qua Waffengewalt, die hier die politische Aussage transportieren. Das Spiel vermittelt - egal ob gewollt oder nicht - somit eine politische Aussage. ${ }^{6}$ Doch lassen sich auch in Spielen wie The Sims, Super Mario Bros. und Destiny 2, die nicht explizit realweltliche politische Akteure in ihre Spielwelt integriert haben, politische Aussagen feststellen? Dies wird im Folgenden näher erläutert.

Über das Politische im digitalen Spiel

Ob Spiele politisch sind, hängt vor allem von der Definition des Politischen ab. In der Politikgeschichte wird dabei im Regelfall auf einen breiteren inklusiven Politikbegriff zurückgegriffen: Solche Ansätze eröffnen uns laut der Historikerin Ute Frevert „den Blick für vielfältige Machtbeziehungen, die sich als politische dort konstituieren, wo es um die Begründung, Verteidigung und Ablehnung ungleicher sozialer Beziehungen geht; [sie entde-

${ }^{4}$ Michelle Erhardt, „The Division doesn't want you to think about 9/11” in killscreen.com $<$ https://killscreen.com/articles/the-division-doesnt-want-you-to-think-about-911/>

5 Aus dem Werbetext: „Alles beginnt am Black Friday, als eine verheerende Pandemie durch die Straßen von New York City schwappt und die Gesellschaft nach und nach ins Chaos stürzt. Du [der/die SpielerIn] bist die letzte Hoffnung der Menschheit: Ein Mitglied der Division, einer Einheit von Schläferagenten, die aktiviert werden, um zu retten, was noch übrig ist" in: Homepage zum Spiel Tom Clancy's: The Division <http://tomclancythedivision.ubi.com/game/de-DE/game_info/index.aspx\#page1>

${ }^{6}$ Pfister, 2018. 
cken] diese Machtbeziehungen in einer symbolischen Praxis, die Sinndeutungen sowohl vorgibt als auch kommunikativ verhandelt. ${ }^{67}$ In diesem Sinne sind digitale Spiele politisch, denn auch aus einer kulturhistorischen Perspektive beschränkt sich das Politische nicht länger auf das Handeln staatlicher Akteure sondern findet ebenso in der Sprache - allgemeiner im Symbolischen - und in der Kommunikation seinen Ausdruck. Auch in der Politikwissenschaft wird in der Regel ein ausgesprochen inklusiver Politikbegriff verwendet. Für Thomas Meyer etwa stellt Politik die „Gesamtheit der Aktivitäten zur Vorbereitung und zur Herstellung gesamtgesellschaftlich verbindlicher und/oder am Gemeinwohl orientierter und der ganzen Gesellschaft zugute kommender Entscheidungen“ dar. ${ }^{8}$ Der Begriff „Macht" wird hier durch „Verbindlichkeit“ ersetzt:

Digitale Spiele sind also immer dann politisch, wenn sie in ihrem Narrativ, in ihrer Ästhetik oder aber in ihrer Spielmechanik gesellschaftliche Machtverhältnisse (bzw. die Legitimierung verbindlichen Handelns) reproduzieren, inszenieren oder auch hinterfragen. ${ }^{\text {? }}$

Eine Suche nach politischen Aussagen in digitalen Spielen ist deshalb sinnvoll, weil Spiele wie Grand Theft Auto V (Rockstar Games: US 2013), The Elder Scrolls V: Sylkrim (Bethesda Game Studios: US 2011) oder Call of Duty: Black Ops 3 (Treyarch u.a.: US 2015) heute - gestützt auf professionelle Vertriebswege und großzügige Marketingbudgets - alleine über die offiziellen Verkäufe - jeweils mehr als dreißig Millionen Menschen erreichen. Immer mehr Menschen verbringen immer mehr Zeit mit digitalen Spielen. Diese sind ein wichtiger Wirtschaftsfaktor und nehmen einen fixen Platz im menschlichen Freizeitverhalten ein. ${ }^{10}$ Der Einfluss digitaler Spiele auf unsere Gesellschaft beschränkt sich aber nicht alleine auf ihre wirtschaftliche

${ }^{7}$ Frevert, 2006, 152-164, 163.

${ }^{8}$ Meyer, 2003, 41.

${ }_{9}^{9}$ Das heißt, um ein Extrem zu nennen, dass selbst an unerwarteter Stelle, also auch in Puzzlespielen wie Bejeweled (PopCap Games: US 2000) usf. potenziell politische (Teil-)Aussagen zu finden sind, wie zum Beispiel eine Spielmechanik die auf einem gesellschaftlich und kulturell eintrainierten Akkumulationsstreben basiert oder eine ebenso unhinterfragte aber immer vorausgesetzte Konkurrenz mit anderen SpielerInnen um den High Score. In weniger abstrakten Spielwelten, die sich eindeutiger an unserer Alltagswelt orientieren wie insbesondere First-Person-Shooter, Rollenspiele, Strategiespiele usf. sind die transportierten politische Aussagen eindeutiger feststellbar.

${ }^{10}$ In den letzten dreißig Jahren hat das vermeintlich ,junge“ Medium alle Alters- und Gesellschaftsschichten durchdrungen, wenn auch die Nutzungsfrequenzen nach wie vor unterschiedlich sind. Die aktuellste "Jugend - Information - Medien" Studie des deutschen
Bedeutung, denn digitale Spiele verändern die Menschen, die sie spielen. Sie sind nicht nur Spielzeug oder Zeitvertreib, als welche sie von Teilen der Öffentlichkeit nach wie vor wahrgenommen werden, sondern reflektieren und konstruieren eben auch Politik, Gesellschaft und Kultur. Sie vermitteln Erfahrungen. Darüber hinaus schaffen viele Spiele über komplexe „Communities" wichtige Teilöffentlichkeiten. Sie sind integraler Bestandteil kollektiver Identitätskonstruktion und vor allem sind sie wertvolle historische Quellen der Gesellschaften, die sie hervorbringen, „denn sie sind wirtschaftliche und kulturelle Produkte konkreter menschlicher Gesellschaften aus einer bestimmten Zeit. Als solche geben sie Auskunft über ihre Entstehungszusammenhänge. “11

Die Verarbeitung von politischen Normen und Weltbildern in Spielen muss nicht notwendigerweise bewusst geschehen sein, sondern ist meist Ergebnis der in Form von Spielwelten verarbeiteten Alltagswelt, denn kein Spiel entsteht in einem sterilen Labor ohne Kontakt zur Außenwelt. Spiele-EntwicklerInnen beziehen ebenso wie AutorInnen ihre Ideen aus ihrer kulturellen und politischen Umgebung. Sie übernehmen Ideen, Darstellungsformen und Erzählstrategien aus anderen Medienformen, verarbeiten persönliche Erfahrungen und lassen sich dabei vom Tagesgeschehen inspirieren. Kulturelle Vorstellungen von Vergnügen und Spannung werden in den Spielcode übersetzt. Das Politische kommt hier bereits in der Darstellung staatlicher Institutionen, wie Regierung, Polizei, Rettung oder Militär ins Spiel. Aber auch im Spielcode angelegte Verhaltensmuster wie „Konkurrenz“ und „Konflikt", moralische Fragen, die Definitionen von „gut" und „böse“ oder

Medienpädagogischen Forschungsverbunds Südwest gibt beispielsweise an, dass knapp $78 \%$ aller deutschen Jugendlichen regelmäßig spielen - nur $9 \%$ spielen gar nicht. In: Medienpädagogischer Forschungsverband Südwest, 2016. Mit dem aktuellen Heranwachsen einer ersten Generation von Menschen, für die der Umgang mit digitalen Spielen von Kindheit an Selbstverständlichkeit war, handelt es sich auch nicht länger um ein Jugendphänomen. Eine amerikanische Studie im Auftrag der Entertainment Software Association beziffert das Durchschnittsalter der SpielerInnen 2015 etwa mit 38 Jahren und mit jedem Jahr wächst der Prozentsatz älterer Menschen, die digitale Spiele spielen. In: Entertainment Software Association, 2016. Solche vereinzelten Erhebungen können uns naturgemäß nur einen ersten Eindruck der Verbreitung digitaler Spiele in unseren Gesellschaften geben, denn umfassende, länderübergreifende Studien fehlen nach wie vor. Auch gilt es festzuhalten, dass sich das Medium zwischenzeitlich soweit diversifiziert hat, dass nicht länger von „einem“ Publikum gesprochen werden kann, sondern vielmehr - wie etwa beim Spielfilm und Roman - korrekter von mehreren Publika zu sprechen wäre.

${ }^{11}$ Köstlbauer/Pfister, 2018, 98. 
Vorstellungen von „Normalität“ und „Devianz“ sind grundsätzlich als politisch zu werten, da es um die Legitimierung von hierarchischen Machtverhältnissen und die Regelung der menschlichen Zusammenarbeit geht. Überall an ihnen und in ihnen finden sich Spuren jener politischen, gesellschaftlichen und/oder kulturellen Diskurse, die ihre Entstehung bedingen. Die hier kommunizierten politischen Aussagen sind im Sinne von Michel Foucaults Diskurstheorie nicht „Abbilder“ einer Realität, sondern konstruieren in Spielen selbst eine ,gesellschaftliche Wirklichkeit“. ${ }^{12}$ Wir haben es hier mit Diskursen zu tun, welche ,also das Sagbare, Denkbare und Machbare [regeln]. Sie organisieren Wirklichkeit." "13 Ihre Sinnhaftigkeit beziehen die Aussagen eines Diskurses dabei aus ihrer Wiederholbarkeit. ${ }^{14}$

Oft sind sich die MedienproduzentInnen vermutlich der unausgesprochenen Konventionen dieser Diskurse, an die sie sich halten, nicht bewusst. ${ }^{15}$ Sie greifen gewissermaßen automatisch auf gewisse populäre Erzählstrategien und ästhetische Traditionen zurück. Zum einen gibt hier das Medium Spiel die Form der Aussagen vor, andererseits folgen die Spiele in Narrativ, Ästhetik und Spielmechanik den diskursiven Regeln des Sag- und Denkbaren. ${ }^{16}$ Zugleich halten sie sich unbewusst an das rhetorische Design des Mediums oder Genres - also an die mediale Form der Aussage - um eine möglichst affektstarke Wirkung bei den SpielerInnen zu erzielen, schließlich handelt es sich um Verkaufsgüter, welche den KonsumentInnen gefallen wollen. ${ }^{17}$ Nicht zu vergessen bei dieser Aufzählung externer Einflüsse sind hier auch die strukturellen Vorgaben des Codes (der Programmiersprache) selbst. Deswegen ist es auch sinnvoll, bei einer Diskursanalyse politischer Aussagen in Spielen nicht so sehr nach den Wertvorstellungen einzelner EntwicklerInnen (AutorInnen) zu fragen, die sich nur sehr schwer festhalten lassen, sondern stattdessen die diskursiven Regeln der politischen Aussagen selbst in den Blick zu nehmen. Umberto Eco sprach in einem vergleichbaren Kontext von der Intention des Textes (intentio operis beziehungsweise intenzione del testo), die als Gegenstück zu der des Autors/der Autorin

12 Sarasin, 2003, 12.
13 Landwehr, 2009 17 .

${ }_{13}$ Landwehr, 2009, 17.

${ }^{14}$ Hoffarth, 2009, 108.

${ }^{15}$ Vgl. dazu etwa den Spielfilm. Siehe Scheuermann 2009, 63.

${ }^{16}$ Landwehr, 2009, 37.

${ }_{17}$ Schrape, 2012, 16 und John Brindle, ,'Real world issues' in games like Deus Ex are there for marketing reasons, not for art" in zam.com http://www.zam.com/article/985/realworld-issues-in-games-like-deus-ex-are-there-for-marketing-reasons-not-for-art (01.02.2017). zu ergründen sei. ${ }^{18}$ Um eine solche Werkintention herauszuarbeiten, ist es notwendig, aus semiotischer Perspektive die Rhetorik der Spiele und aus ikonographischer Perspektive Darstellungsstrategien zu begreifen. Wenn in einem Spiel beispielsweise die stereotype Figur eines korrupten Senators/Bürgermeisters/Präsidenten vorkommt, ist dies nicht automatisch als bewusstes politisches Statement zu werten, sondern kann auch nur der Rückgriff auf ein übliches Genreversatzstück sein. Trotzdem ist die Verwendung einer solchen Figur immer auch eine politische Aussage, die vor allem durch ihre unbewusste Wiederholung an Gewicht gewinnt. Auch Spiele, die zu Unterhaltungszwecken produziert wurden, transportieren somit politische Aussagen. ${ }^{19}$

Häufig „,verstecken“ sich diese politischen Botschaften an der Oberfläche, erscheinen stereotyp und werden weder von den EntwicklerInnen noch von den SpielerInnen hinterfragt. Sie gerinnen derart zu einem Mythos in der Lesart von Roland Barthes. Dieser hatte zwischen 1954 und 1956 in 53 Texten sogenannte „Mythen des Alltags“ analysiert. ${ }^{20}$ Es handelt sich hierbei um eine Sammlung eklektischer kultursemiotischer Betrachtungen zu Waschmitteln, Charlie Chaplin, der Welt des Catchens usf. In jedem Text legte Barthes in vermeintlich unpolitischen Artefakten und Erzählungen verborgene ideologische „Mythen“ offen, ideologische Botschaften, die aber nicht sogleich als solche erkennbar sind. Dabei hatte Barthes naturgemäß noch nicht an digitale Spiele gedacht. Der Mythosbegriff war aber von Anbeginn an kein bestimmtes Medium gebunden, sondern so offen angelegt, dass er potenziell in jedem Kommunikationsprozess gefunden werden kann.

„Der Mythos ist ein System der Kommunikation“

Bezeichnend ist, dass der Mythos nach Roland Barthes nie ein einzelnes Objekt, ein Wort, ein Bild, ein Gedanke oder eine Idee ist, sondern immer ein Symbol-System, das sich aus all diesen Versatzstücken zusammenfügt und ihnen so eine zusätzliche Bedeutung gibt. Barthes Meinung nach läge das Geheimnis seines Erfolgs auch darin, dass der Mythos vermeintlich zu Natur gerinne, das heißt er wird nicht hinterfragt, weil er ,natürlich“ beziehungsweise selbstverständlich erscheine. Erfolgreiche Mythen würden so

${ }^{18}$ Collini, 2004, $14 \mathrm{f}$

19 Vgl. "Computerspielen sind jedoch selbst dann Ideologien eingeschrieben, wenn das nicht den Intentionen der Entwickler entspricht" in Schrape, 2012, 17.

${ }^{20}$ Barthes, 1964. 
ständig mit neuem Material beliefert, sie reproduzierten sich in gewisser Weise selbst. „Die Sache, die bewirkt, dass die mythische Aussage gemacht wird, ist vollkommen explizit, aber sie gerinnt sogleich zu Natur. Sie wird nicht als Motiv, sondern als Begründung gelesen.“21 Ähnlich hat Michel Foucault im Zusammenhang der „performativen Äußerung“" argumentiert, nämlich, dass die Wiederholbarkeit von Signifikanten, erst zulasse, dass diese naturalisiert, das heißt als selbstverständlich eingestuft würden. ${ }^{22}$

Ich habe mich im Folgenden dazu entschieden, Roland Barthes Mythenbegriff zu nutzen, weil er meiner Ansicht nach besonders eindrücklich vermittelt, dass politische Aussagen oft unbewusst reproduziert werden können. Dabei beziehe ich mich vor allem auf seine Fallstudien und weniger auf seinen erst nachträglich geschriebenen Theorietext „Le mythe aujourd'hui““ Davon ausgehend schlage ich deshalb einen an das Medium digitales Spiel und an meine Fragestellung angepassten politischen Mythosbegriff vor, der sich an Barthes Alltagsmythen orientiert (Punkt 1). Zugleich habe ich den Begriff aber für meine Fragestellung konkretisiert (Punkte 2-4):

Der politische Mythos im digitalen Spiel bezeichnet eine politische beziehungsweise ideologische Aussage (mit implizierter Handlungsanweisung), welche den Anschein der Natürlichkeit angenommen hat und deswegen (oft) weder von SpieleentwicklerInnen noch von SpielerInnen als solche begriffen wird. Der Mythos wird entsprechend unbewusst reproduziert und kann im Prozess der medialen Reiteration ${ }^{23}$ - ebenso unbemerkt - seine Qualität auch verändern. Der politische Mythos zeichnet sich dabei durch folgende vier Charakteristika aus:

1. Der politische Mythos ist nie ein einzelnes Zeichen (eine klischeehafte Figur, ein Motiv, ein Symbol, usf.), sondern eine politische Aussage, die sich aus einem System einander gegenseitig verstärkender Zeichen zusammensetzt.

\section{${ }^{21}$ Barthes, 1964, 85.}

${ }^{22}$ Hoffarth, 2009, 49.

${ }^{23}$ Vgl. dazu auch Jacques Derridas Konzept der Iterabilität. Hierzu: „Ein Zeichen ist wiederholbar, es ,erschöpft' sich nicht und kann daher zu ,Iteration Anlaß geben'; es enthält zugleich aber eine Kraft zum ,Bruch mit seinem Kontext,' es ist darin und schließlich stets eine verwirklichte Möglichkeit, die, indem man es mit anderen Zeichen zusammenstellt, auch anders sein kann.“ In: Zorn, 2016, 74. Für den Hinweis auf Derridas IterabilitätsModel möchte ich ganz besonders Andreas Enderlin von der Universität Wien danken!
2. Der politische Mythos ist eine scheinbar unmittelbar einleuchtende politische Aussage. Deshalb wird er oft an mehreren Orten, das heißt in mehreren Spielen beziehungsweise in mehreren Medienformen wiederholt. Durch seine Wiederholung (Reiteration) verstärkt der Mythos den Anschein der „Natürlichkeit“ und wird dergestalt fortan unbewusst reproduziert. ${ }^{24}$

3. Der politische Mythos verschleiert Geschichte in seiner Argumentation. Er zitiert bekannte ästhetische Vorbilder ${ }^{25}$ und überträgt sie auf aktuelle Phänomene ohne dabei historisch spezifisch zu werden. Das heißt er referenziert nicht so sehr „reale“ historische Phänomene selbst, sondern vor allem ihre (nicht weniger reale) ästhetische Hülle. ${ }^{26}$ Er gewinnt seine Überzeugungskraft aus diesem Verweis auf seine vermeintlichen Vorgänger, was wiederum einen Anschein der Natürlichkeit verstärkt.

4. Trotz seiner vordergründig historischen Argumentation ist jeder politische Mythos eindeutig in seiner Aktualität verortbar und kann nur in einem konkreten zeithistorischen Kontext verstanden werden.

Eben weil wir solch politische Mythen wie zum Beispiel den „der Notwendigkeit eines starken Mannes in der Politik“, den „,des unethischen Grosskonzerns“ ${ }^{“ 27}$ den ,der Umweltzerstörung durch Fortschritt“, den „der systeminhärenten Korrumpierbarkeit von PolitikerInnen“, den „der brutalen Menschenmenge ${ }^{\text {‘28 }}$ oder den „der unethischen wissenschaftlichen Experimente mit katastrophalen Folgen ${ }^{\text {‘ } 29}$ bereits in so vielen Filmen, Romanen und Spielen begegnet sind, nehmen wir sie als ,natürlichen“ Bestandteil des medialen Angebots wahr und hinterfragen nicht länger die implizite politische Aussage. Die genannten Beispiele weisen auch bereits darauf hin, dass am Ursprung der Mythosbildung zumeist ein bewusster Akt der politischen

${ }^{24}$ Judith Butler hat von Derrida die Begriffe der Zitathaftigkeit und Iterabilität (siehe vorhergehende Fussnote) übernommen und ausgebaut. Für sie ist das „Subjekt unmittelbar als Produkt der ordnungesgemäßen Zitation und Wiederholung diskursiver Normen gefasst.“ Rosen/Koller, 2012, 91.

${ }^{25}$ Der Mythos bedient sich vor allem bereits bekannter und verbreiteter Inhalte, also insbesondere bei Stereotypen, Tropes (im englischen Sprachgebrauch), Meistererzählungen usf.

${ }^{26}$ Der Mythos bezieht sich nie auf eine Realität außerhalb der medialen Kommunikation sondern immer nur auf eine mediale Realität.

27 Pfister, 2015.

${ }^{28}$ Pfister, 2016b, 198-201.

${ }^{29}$ Pfister, 2017a. 
Kommunikation stattgefunden hat. ${ }^{30}$ Wird ein solcher Mythos aber erfolgreich, das heißt, wird er fortan unbewusst reproduziert, erfordert er nicht länger eine politische Motivation der SpieleproduzentInnen und gehorcht allein den diskursiven Regeln, die es zu erforschen gilt.

Da sich ein politischer Mythos nie auf ein einzelnes Zeichen beschränkt, sondern immer ein System aus Zeichen bildet, ist es auch sinnvoll, auf den drei Ebenen von Narrativ, Spielmechanik und audiovisueller Ästhetik in den Spielen nach den einzelnen Mythen-Bausteinen (Mythemen) zu suchen und diese gesondert aufzuschlüsseln. Durch einen diachronen und synchronen Vergleich mit ähnlichen narrativen, ästhetischen und spielerischen Bausteinen in anderen Medien lassen sich so jeweils ihre konkreten Funktionen rekonstruieren.

\section{Die Realität des digitalen Spiels}

Eine diskursanalytische Untersuchung von Mythen in digitalen Spielen ermöglicht es uns, deren rhetorische und diskursive Wirkweisen besser zu verstehen. Wir können so lernen, zu verstehen, wie ein Mythos argumentiert/funktioniert. Anhand seiner Häufigkeit können wir bis zu einem gewissen Grad auch rekonstruieren, wie erfolgreich ein Mythos war und ist. Was wir auf diesem Wege aber nicht ermessen können, ist, wie er auf den/die EinzelneN wirkt. Die Frage der Wirkung und Rezeption ist zwar nicht notwendiger Bestandteil einer diskursanalytischen Auseinandersetzung mit dem Phänomen, zugleich aber nicht unerheblich, da der Mythos das Funktionieren einer persuasiven Wirkung voraussetzt.

Dass auch in Massenmedien bedeutende politische und kulturelle Transferprozesse stattfinden, ist keine völlig neue Erkenntnis. In eine ähnliche Richtung dachte etwa der Gesellschaftstheoretiker Niklas Luhman: ,W Was wir über unsere Gesellschaft, ja über die Welt, in der wir leben, wissen, wissen wir durch die Massenmedien. ${ }^{131}$ Der Medienwissenschaftler Jürgen Fritz ${ }^{32}$ spricht in diesen $\mathrm{Zu}$ sammenhang von ,wissenskulturellen Transferebenen“ im digitalen Spiel. ${ }^{33}$ Abseits des Erlernens von Handlungsroutinen (zur Meisterung des Spiels) werden auch Faktenwissen und Sprachbilder vermittelt und Denkschemata

30 Vergleiche weiter hinten den Abschnitt zur „Evil Corporation“ und Ridley Scott.

${ }^{31}$ Luhmann, 2009, 9.

${ }^{32}$ Nicht zu verwechseln mit dem gleichnamigen rechtspopulistischen Autor.

33 Zitiert nach Wesener, 2004, 25-27. übernommen. ${ }^{34}$ Bei diesen Schemata handelt es sich um nichts Anderes als kommunizierte und konstruierte Wertvorstellungen, die sich nach Fritz in tiefenpsychologischen Vorgängen übertragen. Wissenstransfers beschränken sich eben nicht nur auf den Bereich des Akademischen und des Lernens aus Lehrbüchern, denn: ,[a]lle Informationen, die einer Person durch herkömmliche Sinneswahrnehmung nicht zugänglich sind, können zwangsläufig nur durch Medien erworben werden ". ${ }^{35}$ Solche Lernprozesse begleiten den Menschen sein Leben lang. Er oder sie lernt von seinen Eltern die Grundlagen der sozialen Interaktion und sich in spezifischen gesellschaftlichen Kontexten (im Caféhaus, im Restaurant, im Aufzug, usf.) richtig zu verhalten. Hier lernt er/sie im besten Fall seine „Rahmungskompetenzen“, die ihm/ihr später ermöglichen, Situationen richtig zu identifizieren und angemessen zu reagieren. ${ }^{36}$ Er oder sie sammelt weiter konkretes Wissen über zwischenmenschliche Beziehungen und Arbeitsabläufe aber auch abstraktes Wissen über das Funktionieren von Wirtschaftssystemen und politischen Prozessen. Zugleich muss er sich in diesen zu verorten lernen und entwickelt so seine Identität.

Er/sie sammelt Wissen, wenn er/sie im Kino einen Spielfilm schaut, wenn er/sie die Tageszeitung liest, aber auch wenn er/sie Computerspiele spielt. Das kann sowohl explizit erfahrenes Wissen sein, auf das er später bewusst zurückgreift, als auch implizites Wissen, auf das unbewusst zurückgegriffen wird. Implizites Wissen sind dabei all jene Informationen, die er oder sie nicht unmittelbar anwenden kann: neu gewonnene Information über angesagte Cafés in der Lower East Side in Manhattan oder Techniken mittelalterlicher Kriegsführung. Solcherart erworbenes Wissen kann sich in den Worten des Pädagogen Stefan Wesener, ,in Teilen oder ganz mit dem Wissen über die Alltagswelt vermischen, vor allen Dingen dann, wenn es in der Wirklichkeit der Alltagswelt zunächst nicht aktiviert und somit keiner Prüfung unterzogen wird. ${ }^{\text {(37 }}$

Jene alltagsfernen Wissensbestände werden im Regelfall aber nicht unkritisch aufgenommen, denn Niklas Luhmann weist darauf hin, dass „wir so viel über die Massenmedien [wissen], daß wir diesen Quellen nicht trauen

34 Die sogenannten „Fact-Ebene“, „Metaphorische Ebene“ und „Dynamische Ebene“ nach Wesener, 2004, 26.

35 Ebenda 32

${ }^{36}$ Ebenda 27.

37 Ebenda 162. 
können“ “ ${ }^{38}$ Das heißt auf digitale Spiele übertragen, dass beispielsweise nicht jede aus einer Weltraumflugsimulation aufgenommene Information unhinterfragt als plausibles Wissen übernommen wird. Einzelne Teilaspekte aus diesen Spielen können aber durchaus als plausibel eingestuft werden, wenn es sich zum Beispiel um kartographische Darstellungen der Milchstraße, das interaktive Erleben des Trägheitsmoments im All handelt, oder das virtuelle Erfahren der Gravitationskraft betrifft. Solche Lernprozesse finden in der einen oder anderen Form in jedem Spiel statt. Hier wird meist unbewusst neues Wissen mit einem bereits bestehenden Wissenstand auf seine Plausibilität hin abgeglichen. Dieses aus Massenmedien bezogene Wissen schließt sich nach Luhmann, ,wie von selbst zu einem selbstverstärkenden Gefüge zusammen.“ Und: „Man wird alles Wissen mit dem Vorzeichen des Bezweifelbaren versehen - und trotzdem darauf aufbauen, daran anschließen müs$\operatorname{sen}^{\text {‘39 }}$ Das gesammelte Wissen funktioniert wie eine Grundierung, wie ein Rahmen für späteres Wissen.

Ein Abgleich auf Plausibilität bürgt naturgemäß nicht automatisch für die Richtigkeit des erworbenen Wissens, wie es die jüngste Debatte um „FakeNews" in aller brutaler Deutlichkeit gezeigt hat. ${ }^{40}$ Umso dringlicher erscheint eine gründliche Auseinandersetzung mit jenen bisher unbeobachteten Wissenstransfers.

Von derpolitischen Sozialisierung und Kultivation der SpielerInnen

Eine methodische Auseinandersetzung mit diesem Phänomen ist nicht gänzlich neu und wurde in den vergangenen Jahren unter anderem von PolitologInnen, Kommunikations- und MedienwissenschaftlerInnen und SoziologInnen angesprochen: In der Kommunikationswissenschaft wird deshalb mittels Inhaltsanalysen von Spielen einer „sozialen Wirklichkeit“ nachgegangen, die „kognitive Spuren in den Wertvorstellungen ihrer Nutzer hinterlässt“ und so zum Beispiel auch „moralische Werturteile“ beeinflussen könnte. ${ }^{41}$ Der Kommunikationswissenschaftler Christoph Klimmt spricht in diesem Zusammenhang explizit von „Kultivationseffekten“, wenn es um das Übernehmen von Weltbildern in Spielen geht. Darüber hinaus haben Ergebnisse der „narrative persuasion theory“ gezeigt, dass auch Einstellungen über digitale Spiele beeinflusst werden können: „Gerade die Fiktionalität

${ }^{38}$ Luhmann, 2009, 9.

${ }^{39}$ Ebenda.

${ }^{40}$ Ich danke Andreas Enderlin für den Hinweis.

${ }^{41}$ Klimmt, 2009, 68 von Unterbaltungsangeboten (₹u denen grundsätalich auch Computerspiele ₹äblen müssen) [stellt] ein Einfallstor für persuasive Medienwirkungen dar, unter anderem weil die Rezipient/inn/en gar nicht auf einen medialen Persuasionsversuch eingestellt sind und entsprechend keine einstellungsverteidigende kognitive Haltung einnehmen. " ${ }^{* 2}$ Hier zeigen sich in aller Deutlichkeit die Berührungspunkte zum politischen Mythos, der seine Überzeugungskraft aus der Beiläufigkeit schöpft.

Auch im Bereich der Soziologie gehen Untersuchungen davon aus, dass das Erlernen gesellschaftlicher Normen und Regeln nicht nur im Elternhaus, der Ausbildung und im sozialen Umfeld stattfindet, sondern auch mittels der Medien. ${ }^{43}$ Bereits in den Überlegungen des Soziologen Emile Durkheim spielten neben der „methodischen Sozialisation“ (i.e. Erziehung) „,beiläufige und weitgehend unbewusste Lern- und Anpassungsprozesse" eine zentrale Rolle. ${ }^{44} \mathrm{Im}$ Rahmen kritischer Modernisierungstheorien wird des Weiteren die These vertreten, dass die tradierten sozialen Institutionen und damit die sozialen Einbindungen der Einzelnen in westlichen Gesellschaften in den letzten Jahrzehnten an Kraft und Verbindlichkeit verloren hätten, wodurch die Mediensozialisation zusätzlich an Bedeutung gewonnen hätte. ${ }^{45}$ Allerdings sei es schwierig bis unmöglich die unterschiedlichen Sozialisierungsinstanzen (Familie, Schule, Peer Group, Medien usf.) voneinander zu trennen, da Medien alle anderen Lebensbereiche durchdringen. ${ }^{46}$ Die Frage der Transferprozesse beziehungsweise der Medienwirkung in digitalen Spielen befindet sich dabei nach wie vor im Anfangsstadium und empirische Studien, welche die Theorien anhand digitaler Spiele belegen und ausführen, bleiben die Ausnahme, beziehungsweise beschränkten sich bisher vor allem auf die Frage möglicher Auswirkungen im Hinblick auf aggressives Verhalten und Gewaltverbrechen. ${ }^{47}$ Es wäre allerdings notwendig Untersuchungen der politischen, gesellschaftlichen und kulturellen „Wirkung“ von digitalen Spielen nicht alleine auf die Frage der Gewaltbereitschaft zu reduzieren,

42 Ebenda, Vergleiche auch Fromm /Biermann, 2009, 120: „Es kann ang.enommen werden, dass eine Distanzierung von derart beiläufig vermittelten „heimlichen“ Botschaften schwieriger ist als von expliziten Inhalten.

${ }^{43}$ Schulz, 2011, 165 und Fromme/Biermann, 2009, 115.

44 Ebenda 113.

45 Ebenda $114 f$

46 Ebenda 117.

${ }^{47}$ Klimmt, 2009, 69 und Pfister, 2016c. 
sondern allgemein die Konstruktion von Machtbeziehungen und Weltbildern in Spielen zu hinterfragen. ${ }^{48}$ An dieser Stelle ist deshalb eine ergänzende kulturhistorische Perspektive notwendig. Die Suche nach politischen Mythen in digitalen Spielen ermöglicht dabei zwar keine Aussagen über die Wirkung auf die einzelnen SpielerInnen, sie ermöglicht uns aber sehr wohl eine genaue Analyse der Wirkmechanismen und Argumentation der politischen Aussagen in Spielen. Die Anwendung dieser Theorie funktioniert hier „so ähnlich wie in der Mikroskopie ein Färbemittel bei der Betrachtung von Proben: Ihr Einsatz macht gewisse Elemente und ihre Zusammenhänge erst sichtbar. ${ }^{\text {(49 }}$

\section{Politik spielend erkennen lernen}

Wenn heute in digitalen Spielen Machtbeziehungen kommuniziert, konstruiert und perpetuiert werden, handelt es sich nicht um eine Eigenheit des Mediums, sondern um einen ursprünglichen Prozess politischer und kultureller Reproduktion, der sich über alle Medien hinweg feststellen lässt. Dies gilt demgemäß auch für die zu untersuchenden politischen Mythen, die mühelos vom Film zum Spiel zum Roman usf. wechseln. Sie imaginieren und/oder spiegeln dabei bestimmte Wertesysteme oder versuchen - im Ausnahmefall - bewusst dominante Diskurse aufzubrechen.

Es gibt durchaus Spiele, die ganz bewusst eine politische Aussage transportieren. Das sind zum Beispiel die gesellschaftskritischen „Culture Jamming“ Projekte des italienischen Künstlers Paolo Pedercini (Molle Industria) sowie die Spiele der österreichischen Künstlergruppe gold extra. Im Spiel Unmanned (Molle Industria: I 2012) wird beispielsweise ein Tag im Leben eines Drohnenpiloten simuliert, im Mod Frontiers (gold extra: A 2012) versucht man im perspektivischen Rahmen eines First-Person Shooters als afrikanischer Flüchtling die Grenzzäune von Ceuta zu überwinden. ${ }^{50}$ Offen politisch sind im Normalfall auch jene didaktischen Spiele, die im Auftrag politischer Akteure wie Ministerien, Parteien und NGOs entwickelt wurden. Oft werden diese als Serious Games bezeichnet, weil die Vermittlung eines ernsthaften Anliegens im Vordergrund steht. Diese Spiele wollen die SpielerInnen politisch überzeugen und erziehen und benutzen bewusst die rhetorischen Mittel des Mediums Spiel für ihre Zwecke, wie es der deutsche

\footnotetext{
${ }^{48}$ Ebenda.

${ }_{49}$ Nach Leitgeb, 2008, 11.

${ }^{50}$ Pfister, 2016a, 186f.
}

Medienwissenschaftler Niklas Schrape in seiner Untersuchung dargestellt hat. $^{51}$

Die Reichweite solcher Spiele bleibt im Normalfall begrenzt. Für eine Analyse breitenwirksamer politischer Kommunikationsprozesse in Spielen ist es deshalb notwendig, sich die Spiele anzuschauen, die ein größeres Publikum erreichen, also jene auf Hochglanz polierten Spielen, die sich millionenfach verkaufen und im Jargon der Branche Triple-A-Games genannt werden. Im Ausnahmefall findet man auch hier EntwicklerInnen, die sich bewusst mit politischen Themen auseinandergesetzt haben. Prominentestes Beispiel ist der First-Person-Shooter BioShock (2K Games: US 2007), in welchem der leitende Entwickler Ken Levine mit der fiktiven Unterwasserstadt „Rapture" bewusst die Dystopie einer Gesellschaft erschaffen hat, die auf den politischen Gedanken Ayn Rands beruht. Bildgewaltig werden hier die Folgen eines entfesselten Libertarismus gezeigt. ${ }^{52}$ Auch für das Action-Adventure Beyond Good and Evil (Ubisoft: F 2003) gab der leitende Entwickler Michel Ancel politische Motive an: Das Spiel dürfe als Reaktion auf die Anschläge vom 11. September und das anschließende „fear climate“ [sic] verstanden werden. ${ }^{53}$ Solch offene Stellungnahmen zu den politischen Hintergründen eines Spiels bleiben aber die Ausnahme. Weitaus häufiger findet eine politische Kommunikation in Spielen unbewusst in Form von Alltagsmythen statt.

\section{Von bösen Großkonzernen und wahnsinnigen WissenschaftlerInnen}

Was politische Mythen von anderen politischen Botschaften in digitalen Spielen unterscheidet ist, dass es sich immer um Zeichensysteme handelt und, dass sie immer wieder und an mehreren Orten auftauchen. Man erkennt sie entsprechend am Rückgriff auf vermeintliche Stereotype oder Gemeinplätze. Exemplarisch dafür ist der Mythos einer „Evil Corporation“. Eine solche würde, so argumentiert der Mythos, alleine motiviert durch Gewinnstreben keinerlei Rücksicht und ethische Bedenken kennen. Die Inszenierung menschenverachtender Großkonzerne als Antagonisten im Hintergrund findet vor allem in Spielen mit einem Science-Fiction- und/oder Horror-Setting Gebrauch: die Umbrella Corporation in der Resident-Evil-Serie

\footnotetext{
${ }^{51}$ Schrape, 2012.

52 Gatterbauer/Mikolasch, 2016

53 Purchese, Robert: "BG\&E2 inspired by September 11. Plus politics and current affairs in general.” In eurogamer.net. < http://www.eurogamer.net/articles/ancel-thinks-zelda-is-
} out-of-touch>. 
(Capcom: J 1996 usf.), Phenotrans in Dead Rising (Capcom: J 2006), Abstergo in Assassin's Creed (Ubisoft Montreal: CA 2007), Hyperion in Borderlands (Gearbox Software: US 2009), usf. usf.

Die „Evil Corporation“ muss dabei in Spielen nicht mehr umständlich als solche eingeführt werden, weil SpielerInnen diese dank der häufigen Wiederholung sofort erkennen (bzw. im Sinne von Foucault lesen) und entsprechend als Widersacherin identifizieren können. Dieses sofortige Verstehen ohne Erklärung ist notwendige Voraussetzung für den Mythos, es ist sein Anschein der Natürlichkeit. In diesem konkreten Fall wurde der Mythos bereits zuvor im Science-Fiction Film eingeübt: Cyberdyne (TerminatorReihe), OmniCorp. (Robocop-Reihe), die Tyrell Corporation (Blade Runner) und natürlich Weyland Yutani (Alien-Reihe). Die letztgenannte „Evil Corporation" vollzieht dabei mühelos die Übersetzung von einem Medium ins andere, wie es zuletzt im Spiel Alien: Isolation (Creative Assembly: GB 2014) ausgeführt war. Dieses Beispiel zeigt auch eindrucksvoll, wie Mythen im Medium digitales Spiel durch das Zusammenwirken mehrerer Mytheme eine neue Qualität bekommen. Neben der narrativen und audiovisuell-ästhetischen Inszenierung, wird der Mythos hier auch mittels der Spielmechanik aufgebaut. Im Fall von Alien: Isolation beispielsweise, wird durch eine hohe Spielschwierigkeit, seltene Speichermöglichkeiten und eine unvorhersehbare „Künstliche Intelligenz“ das Gefühl der Ohnmacht im Angesicht der „Evil Corporation“ bei den SpielerInnen noch verstärkt. Dies spiegelt sich im narrativen Aufbau, denn die SpielerInnen bekommen in der Erzählstruktur des Spiels gar nicht erst die Möglichkeit, gegen die Weyland Yutani Corporation anzukämpfen; ihre einzige Hoffnung ist die Flucht. Die „Evil Corporation" mag hier wie ein simples Requisit aus dem Science-FictionGenre erscheinen, sie hat ihren Ursprung aber in einer genuin politischen Angst, die auch immer in einem zeithistorischen Kontext zu lesen ist. In diesem Fall gibt Ridley Scott, Regisseur des ursprünglichen Alien-Films, selbst Auskunft: "It's possible that the value of humans could have diminished. I'm now thinking on the level of the Big Brother idea of a lifeless mega structure and its attitude toward human employees, who are considered expendable. In this instance, the machinery, information data, and cargo are of more importance to corporations than the individuals on their ships. I certainly think this situation has parallels today. ${ }^{654}$

\footnotetext{
54 Scott, 2005, 45f.
}

Ein zentrales Motiv des Science-Fiction-Genres war schon immer das Aufzeigen und Verarbeiten kollektiver gesellschaftlicher Ängste: „Eine glaubhafte und faszinierende Zukunft zu entwerfen, steht [...] erst an zweiter Stelle. ${ }^{\text {“55 }}$ Der transmediale politische Mythos verweist in unserem Fall implizit auf medial weltweit verbreitete Skandale wie zum Beispiel die unethischen Verkaufsmethoden von Nestlé für Babynahrung in Entwicklungsländern in den 1970er-Jahren ${ }^{56}$ oder die durch Kosteneinsparungen verursachte Explosion der Ölbohrplattform Deepwater Horizon 2010 ${ }^{57}$. Hier treten die potenziellen Transferprozesse von Weltbildern deutlich zu Tage: Wenn wir also heute in der Berichterstattung mit Bildern eines Brandes in einem Sweatshop in Ostasien konfrontiert werden, ist unsere Erwartungshaltung oft bereits vom medialen Kontakt mit der einen oder anderen „Evil Corporations" grundiert worden. Zugleich hat der Mythos aber in den meisten Fällen die ursprünglich bewusste Kritik eingebüßt.

Neben der häufigen Wiederholung zeichnen sich politische Mythen durch einen starken Rückgriff auf vermeintlich historische Argumente und Bildstrategien aus. Denn der Eindruck der Natürlichkeit wird auch durch eine starke Referenzierung historischer Vorbilder und Vorgeschichten erreicht. Durch den impliziten Verweis auf vergleichbare Mythen und ihre Symbole erhöht jeder politische Mythos seine Plausibilität. Zur Illustration dieses Gedankens hilft ein Blick auf den politischen Mythos der ,unethischen Experimente mit katastrophalen Folgen“. Auch dieser kommuniziert, ungeachtet seiner oft fast schon karikierenden Überzeichnung verrückter WissenschaftlerInnen, grundsätzliche politische Bedenken, wenn es im Kern um die Frage der ethischen Grenzen der Forschung geht. Im Rollenspiel Fallout (Black Isle Studios: US 1997) beispielsweise müssen die SpielerInnen einen namenlosen Wissenschaftler besiegen, der mittels eines „Forced Evolutionary Virus“ Menschen großflächig zu „Supermutanten“ umformen/verbessern will. Trotz der überzeichneten Form bleibt die Frage die gleiche: Wo sind die Grenzen der Zulässigkeit wissenschaftlicher Experimente anzusetzen? Eine Frage, die uns schon seit längerer Zeit begleitet, denn der (zumeist männliche) verrückte Wissenschaftler kann auf eine lange historische Tradition zurückblicken. Er verweist mehr oder weniger direkt auf Vorläufer

55 Gözen, 2012, 267.

56 „Brust statt Schnuller“ in Der Spiegel 23/1981, 150f. <http://www.spiegel.de/spiegel/print/d-14336212.html>

${ }^{7}$ Zum Beispiel Ian Urbina, Documents Show Early Worries About Safety of Rig in nytimes.com, 29.05.2010, <http://www.nytimes.com/2010/05/30/us/30rig.html?hp> 
wie „Dr. Jekyll“ (Robert Louis Stevenson 1886), „Dr. Frankenstein“ (Mary Shelley 1818) und „Dr. Faustus“ (Christopher Marlowe 1587). Zugleich ermöglicht uns das genannte Beispiel auf die starke historische Verankerung im zeithistorischen Kontext eines jeden Mythos hinzuweisen, denn trotz seines oft generisch wirkenden Erscheinens - oder gerade deshalb - eignet sich der Mythos besonders gut zur Untersuchung einer öffentlichen Wahrnehmung historischer politischer und gesellschaftlicher Debatten. War es bei Faust noch ein Pakt mit dem Teufel, bei Dr. Frankenstein die Medizin und bei Dr. Jekyll die (Al-)Chemie, ist es heute in Fallout und vergleichbaren Spielen die Gentechnik. Aus diesen Gründen muss eine Untersuchung politischer Mythen neben einer narratologischen, ludologischen und ikonographischen auch immer eine kulturhistorische Perspektive einnehmen, denn politische Mythen sind sowohl gegenwartsbezogene Phänomene, die sich aber auch immer in eine historische Kontinuität einschreiben, weswegen neben der synchronen Kontextualisierung der Mythen auch immer eine diachrone zwingend notwendig ist. ${ }^{58}$

\section{Scbluss}

Wenn wir - aus einer strukturalistischen Perspektive heraus - davon ausgehen, dass politische Weltbilder nichts „Natürliches“ an sich haben, also keine Existenz außerhalb ihrer diskursiven Funktion haben, ist es notwendig, dem Prozess ihrer Konstruktion überall dort nachzuspüren, wo er sich manifestiert, um ihn umfassend begreifen zu können. Hierbei ist es im Grunde gleichgültig, ob wir nach kollektiven Identitäten, Sozialisierungsoder Kultivationsprozessen oder nach politischen Mythen suchen. Solange wir davon ausgehen können, dass der zu untersuchende Endzustand, also ein spezifisches Weltbild oder -verständnis, mit Ergebnis eines Kommunikations- und Konstruktionsprozesses ist, muss es Ziel der Geistes- und Sozialwissenschaften sein, diesen Konstruktionsprozess gründlich zu verstehen. Dieser findet immer im Rahmen historischer Diskurse statt, welche die Rahmenbedingungen vorgeben. In digitalen Spielen - und zwar auch in jenen, die als reine Unterhaltungsprodukte wahrgenommen werden - können potenziell Sozialisierungsprozesse stattfinden. In digitalen Spielen werden Machtverhältnisse legitimiert und verbindliche Verhaltensnormen und Regeln konstruiert. Dies geschieht, ebenso wie auch in anderen populärkulturellen Medien, zu großen Teilen unbemerkt und sollte deshalb dringend

${ }^{58}$ Zum Mythos Mad Scientist vgl. auch Pfister, 2017a. durch anschauliche und gut vermittelbare Forschungsergebnisse einer wissenschaftlichen, politischen und gesellschaftlichen Öffentlichkeit sichtbar gemacht werden, um objektiv diskutiert werden zu können.

Eine gründliche Untersuchung politischer Mythen in digitalen Spielen würde zu einem besseren Verständnis der oft unsichtbaren Genrekonventionen und Spielregeln verhelfen und somit zu einem besseren Verständnis digitaler Spiele führen. Auch ermöglicht eine gründliche Analyse eine neue Perspektive auf zeitgenössische politische Diskurse, deren Rahmen und Grenzen von diesen politischen Mythen mitbestimmt werden. Politisch sind Spiele immer dann, wenn Machstrukturen und verbindliches Handeln einer Gemeinschaft in Spielen kommuniziert werden, konkret wenn Wertungen transportiert werden, die allgemeine Gültigkeit für sich in Anspruch nehmen, wie zum Beispiel die Tabuisierung von Angriffskriegen ${ }^{59}$ oder die Heroisierung der Figur des einsamen Helden in der Politik.

Schließlich gilt es, mit dem Irrglauben, Spiele könnten apolitisch sein, aufzuräumen. Er nimmt seinen Ursprung in einem ausgesprochen exklusiven aber auch negativen Politikverständnis und geht mit dem Generalverdacht der unlauteren politischen Propaganda (im Grunde selbst ein politischer Mythos?) einher. Politik wird immer mehr als ein Fremdkörper verstanden und nicht länger als natürlicher Bestandteil unseres Alltages. Eine gründliche Diskursanalyse digitaler Spiele könnte hier helfen nachzuweisen, dass politische Diskurse normale Bestandteile unserer Alltagswelt und an sich weder positiv noch negativ zu werten sind. Durch ein besseres Verständnis bisher unbeobachteter rhetorischer Mittel kann vielleicht die Grundlage für fruchtbare Diskussionen sowohl in der Forschung als auch im Schulunterricht geschaffen werden.

\section{Literatur}

Barthes, Roland (1964): Mythen des Alltags, Suhrkamp, Frankfurt a. M.

Collini, Stefan (2004): Introduzione in: Eco, Umberto, Interpretazione e sovrainterpretazione, Bologna, 5-29.

Entertainment Software Association (Hg.) (2016): Sales, Demographic and Usage Data. Essential facts about Computer and Video Game Industry, in: $<$ http://essentialfacts.theesa.com/Essential-Facts-2016.pdf $>$.

\footnotetext{
59 Pfister, 2017b.
} 
Frevert, Ute (2006): Neue Politikgeschichte, in: Eibach, Joachim/ Lottes, Günther (Hg.), Kompass der Geschichtswissenschaft, Göttingen, 152-164.

Fromme, Johannes/Biermann, Ralf (2009): Identitätsbildung und politische Sozialisation in: Bevc, Tobias/Zapf, Holger (Hg.), Wie wir spielen was wir werden. Computerspiele in unserer Gesellschaft, Konstanz 2009, 113-138.

Gatterbauer, David/Mikolasch, Ulrich (2016): Von Entrückung und mythischer Morgenröte: Utopien und Dystopien in The Elder Scrolls IV: Oblivion und BioShock - ein Vergleich in: Spiel-Kultur-Wissenschaften, <http://spielkult.hypotheses.org/981>.

Gözen, Jiré Eminé (2012): Cyberpunk Science-Fiction. Literarische Fiktionen und Medientheorie, Bielefeld.

Hoffarth, Britta (2009): Performativität als medienpädagogische Perspektive, Bielefeld.

Klimmt, Christoph (2009): Empirische Medienforschung: Kommunikationswissenschaftliche Perspektiven auf Computerspiele, in: Bevc, Tobias/Zapf, Holger (Hg.), Wie wir spielen was wir werden. Computerspiele in unserer Gesellschaft, Konstanz, 65-74.

Köstlbauer, Josef (2013): Editorial. In: Computer - Spiele - Geschichte. Historische Sozialkunde 4/2013.

Köstlbauer, Josef/Pfister, Eugen (2018): Vom Nutzen und Nachteil einer Historie digitaler Spiele in: Hust, Christoph (Hg.), Digitale Spiele Interdisziplinäre Perspektiven zu Diskursfeldern, Inszenierung und Musik, Bielefeld, 89-106.

Landwehr, Achim (2009): „Historische Diskursanalyse“, Frankfurt a. M.

Leitgeb, Christoph (2008): Barthes` Mythos im Rahmen konkreter Ironie, München.

Luhmann, Niklas (2009): Die Realität der Massenmedien, Wiesbaden.

Medienpädagogischer Forschungsverband Südwest (Hg.) (2016): Jugend-, Information-, (Multi-)Media . Basisstudie zum Medienumgang 12- bis 19-Jähriger in Deutschland, Stuttgart.

Pfister, Eugen (2015): „Von Xenomorphs und anderen Raubtier(kapitalist)en, In: Spiel-Kultur-Wissenschaften, <http://spielkult.hypotheses.org/140>.

Pfister, Eugen (2016a): „A passport is required“ - Imaginationen von Grenzen und Flucht im digitalen Spiel, In: Breitenfellner, Helene/Crailsheim, Eberhard/Köstlbauer, Josef/Pfister, Eugen (Hg.), Grenzen - Kulturhistorische Annäherungen, Wien, 81-197.
Pfister, Eugen (2016b): „Des patriotes, ces abrutis!“ Imaginationen der französischen Revolution im digitalen Spiel Assassin's Creed: Unity, In FrühneuzeitInfo 27/2016, 198-201.

Pfister, Eugen (2016c): Plädoyer für eine argumentenbasierte Debatte zur Wirkung gewalthaltiger Spiele, In: Spiel-Kultur-Wissenschaften, $<$ http://spielkult.hypotheses.org/1114>.

Pfister, Eugen (2016d): „Well, it’s definitely creepy down here“ Das Politische im Teenie-Slasher-Genre, In: Spiel-Kultur-Wissenschaften, $<$ http://spielkult.hypotheses.org/618>.

Pfister, Eugen (2017a): „Doctor nod mad. Doctor insane.“ - Eine kurze Kulturgeschichte der Figur des mad scientist im digitalen Spiel, In: Görgen, Arno/Inderst, Rudolf (Hg.) PAIDIA-Sonderausgabe: Die Darstellung von Wissen-schaft, Forschung und Technologie in digitalen Spielen (27.07.2017).

Pfister, Eugen (2017b): „Don’t get mad, get even.“- Der Opfermythos in digitalen Spielen, In: Spiel-Kultur-Wissenschaften, <http://spielkult.hypotheses.org/1497>.

Pfister, Eugen (2018): „Keep yopur Politics out of my Games!“, in: Spiel-KulturWissenschaften, <http://spielkult.hypotheses.org/1566>.

Rosen, Nadine/Koller, Hans-Christoph (2012): Interpellation - Diskurs - Performativität in: Norbert Ricken und Nicole Balzer, Judith Butler: Pädagogische Lektüren, Wiesbaden, 75-94.

Sarasin, Philipp (2003): Geschichtswissenschaft und Diskursanalyse, Frankfurt am Main.

Scheuermann, Arne (2009): Zur Theorie des Filmemachens. Flugzeugabstürze, Affekttechniken, Film als rhetorisches Design, München.

Schrape, Niklas (2012): Die Rhetorik von Computerspielen. Wie politische Spiele überzeugen, München.

Schulz, Winfried (2011): Politische Kommunikation. Theoretische Ansätze und Ergebnisse empirischer Forschung, Wiesbaden.

Scott, Ridley (2005); interviewt von Danny Pery in: Knapp, Laurence F./Kulas, Andrea F. (Hg.), Ridley Scott Interviews, Jackson.

Wesener, Stefan (2004): Spielen in Virtuellen Welten. Eine Untersuchung von Transferprozessen in Bildschirmspielen, Wiesbaden.

Zapf, Holger (2009): Computerspiele als Massenmedien. Simulation, Interaktivität und Unterhaltung aus medientheoretischer Perspektive, in: Bevc, Tobias/Zapf, 
Holger (Hg.), Wie wir spielen was wir werden. Computerspiele in unserer Gesellschaft, Konstanz, 11-26.

Zorn, Daniel Pascal (2016): Vom Gebäude zum Gerüst. Entwurf einer Komparatistik reflexiver Figurationen in der Philosophie, Berlin 2016,

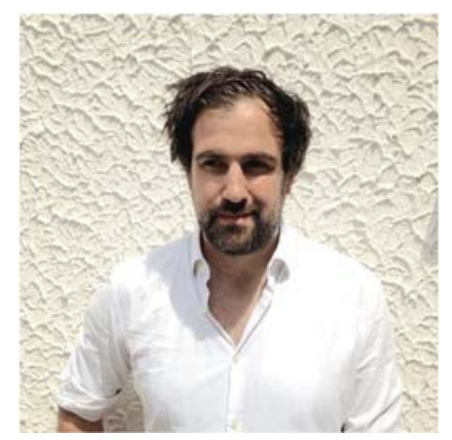

Eugen Pfister ist Historiker und Mitbegründer des Arbeitskreises Geschichtswissenschaft und

Digitale Spiele (gespielt.hypotheses.org). Seit 2018 leitet er das SNF-Ambizione Forschungsprojekt „Horror-Game-Politics - die Rhetorik des Grauens" an der Hochschule der Künste Bern - HKB. Er hat Geschichte und Politikwissenschaft in Wien und Paris studiert und zur Geschichte der Politischen Kommunikation an den Universitäten Frankfurt a.M. und Trento promoviert. Seine Forschungsschwerpunkte sind Spielgeschichte, Ideengeschichte und Geschichte der politischen Kommunikation. Seit 2015 betreibt er einen Forschungsblog "SpielKultur-Wissenschaft. Mythen im Digitalen Spiel" (Spielkult.hypotheses.org).

Kontaktadresse: eugen.pfister@univie.ac.at Spiele im Diskurs. URL: http://www.medien-im-diskurs.de

cc) (†) $\odot$ Inhalt steht unter einer Creative Commons Namensnennung-NichtKommerziell-KeineBearbeic) tung 3.0 Unported-Lizenz. 